\title{
Physical Interface for Robotic Marionette Camera (RMC): Hardware Controlling Platform for Robotic Videography Motion Design
}

XXIV International Conference of the Iberoamerican Society of Digital Graphics Medellin | Colombia

\author{
Ebrahim Poustinchi \\ Kent State University | United States | mpoustin@kent.edu \\ Mona Hashemi \\ Kent State University | United States | mhashemi@kent.edu \\ Cory Krivanek \\ Kent State University | United States | ckrivan1@kent.edu
}

\begin{abstract}
This project-based research is an investigation on controlling robotic videography/camera through an interactive physical interface. Here referred to as Robotic Marionette Camera (RMC), this research project is enabling designers and videographers to design precise robotic videography scenarios and camera-paths in the physical world with similar qualities to the digital design environments.
\end{abstract}

Using the ideas of a "digital" camera in design software platforms, RMC looks at concepts such as aiming, zooming in and out, panning, orbiting, and other motions/operations borrowed from cinematography, such as tilting, rolling and trucking amongst others.

As a physical/hardware interface, RMC enables real-time interaction with an industrial robot arm through a custom-made hardware controller. Using a tangible interface, RMC users can design, edit, and program the robotic videography paths interactively without a need for programming knowledge.

Keywords: Robotics, Design, Interface Design, Videography, Human-Robot Interaction

\section{INTRODUCTION}

It took around twenty years for the early CNC machines to change into five-axis robot arms that we generally classify as industrial robots. George C. Devol and Joseph Engelberger first introduced early robots to industrial manufacturing in the 1950s and early 1960s. (Nof, 1999) These robots played an essential role in mass production industries until the late 1980s when Japanese companies started to investigate their performance in large-scale construction. However, it took until early 2000 , when the use of robotics was affected by the new shift in architecture paradigm. In the age of computational movement and digital design tools, the complexity of design solutions demanded more digitally controlled manufacturing systems (Budig et al., 2014).

Moving forward, using industrial robots in the design framework and making them accessible for creative industry rather than mass production and manufacturing, attracted a wide range of attention from architects and designers (Braumann \& Sigrid, 2011) (Gramazio \& Kohler, 2008). These robots, combined with digital programming and custom made tools attached to them, have created the opportunity for designers to explore possibilities of enriching the physical nature of architecture (Gramazio, Kohler, \& Willmann, 2008).

In recent years, many research investigations explored the possibilities of integration between computational design and digital fabrication (Retsin \& García, 2016) (Yunis et al., 2014). Some academic institutes started using humanrobot interaction criteria for designing a "Collaborative Construction" workflow (Vasey et al., 2016). Based on these innovations, the process of robotic manufacturing and robotic assembly enters into a new realm of humancomputer (robot) interaction.

However, robotics is not limited to its conventional use for manufacturing and fabricating. It has evolved impressively in other disciplines and acted as a medium to represent and illustrate artistic outcomes. In his book, Robot House, Peter Testa proposes design and imaging techniques, using robotics, machine vision, and augmented reality for designers, artists, and filmmakers (Testa, 2017). Another non-productional robotic project is RAI Alpha that studies the possibilities of imaging and robotically- controlled perspective as possible architectural tools and potential mediums for design explorations (Poustinchi, 2019). These developments show that robots can be used actively as creative design tools in different design-related categories.

Consequently, a tendency was created among designers, artists, and architects toward using the visual opportunities of robots. They started to look into possibilities of using robots not necessarily as a making tool to build stuff physically, but as an active agent to augment the experience on top of space. Bot \& Dolly, a creative robotic studio specialized in automation, robotics, and filmmaking, 
was at the heart of this development. This studio has introduced many unique projects, including the special effects for the film Gravity (URL1) or the BOX project, a physical installation augmented with projection-mapping (URL2).

Another example of innovative companies in robotic experiments is Motorized Precision (URL2). This professional studio develops simple, easy-to-use, and intuitive motion control. The robot is an integral part of their projects, creating spatial effects in commercials, films, and visual effects videos. KIRA is their new high-speed six-axis cinema robot. Running with MPStudio software, designed and developed by the company, this robot can perform unmatched, fast, and creative motions (URL3).

Recently, there have been several software developments to incorporate robots into the design workflow and provide interfaces that help designers work in visual programming environments and control and simulate the robot behavior like KUKA RPC (Braumann \& Brell-Cokcan 2012) and HAL (Schwartz 2013). In addition, there have been other research investigations on creating software and plug-ins to work with robotic motions interactively. Oriole is a parametric tool and a custom-made plug-in for Grasshopper 3D used for robotic videography that allows designers to precisely simulate the robot's motion and path (Poustinchi, 2019). This user-experience platform provides the designer with the opportunity to document the physical experience of their design and actively engage the audience in their experiments.

Due to these recent influential works, robots gradually started to secure their place as cutting-edge design research tools in architecture and research centers. However, all these experiments with robots require digitally skilled users with some digital design background and software knowledge. The high price of related software packages is another limiting factor resulting in lower communication between humans and robots. Therefore, there was a missed opportunity to explore the performative and creative aspect of the robot through a custom-made, user-friendly tool. The tangible controller for controlling the motion of the ABB-IRB 140 industrial robot by Andrew Payne (Payne, 2011) is one example of these intuitive, collaborative platforms.

In the light of the previous research investigation, robots have found their way in the creative design, representation, and visual art disciplines, including videography. Motion in space is a tangible phenomenon, and videography can be understood as an experimental process. A user--motion designer, would rather experience a series of actions or steps that lead to the final output than digitally entering the codes or inputs through software. Considering this issue and following the increased improvements in the design and invention of user-friendly applications and devices to control complex digital tools, RMC seeks to provide a sufficient medium for designers/architects/users to use robots for the robotic videography, through a custom-made physical interface.

Building upon earlier investigations in the field of humanrobot interaction, robotic videography, RMC research is developed around three main themes:
1- Translating the possibilities and flexibilities of a digital camera-from design software platforms, into the physical world.

2- Developing a workflow for designers and artists to be a bale to use the power of robotics and digital design through a physical interface way and with no programming skill required.

3- Exploring the possibilities of a customized physical/ hardware interface, as a new way to adopt cutting edge technologies in creative fields.

\section{METHODOLOGY}

As project-based research, RMC aims to simplify the complex process of programming a robot for videography by physicalizing some of the programming steps of the process through the interactive use of a tangible controller. Tested as part of the design studio at Kent State University, different users with different design and technology backgrounds used RMC - as a platform to design/rebuild a digital camera, in the physical world through the use of UR10 robot arm.

As an experimental setup and through enabling the realtime communication between the UR-10 robot arm, and the physical controller-RMC hardware, this research project, investigates the impact of this new method of interaction on possible design projects.

Although it is not the aim of this research to study all the possible effects of RMC on the process of robotic videography, it is intended to report on the qualitative and conceptual advantages of RMC in a comprehensive manner.

Testing the RMC platform, in the setup of the Robotically Augmented Design (RAD) Lab at Kent State University, it was intended to mainly focus on how users interact with the RMC platform, and how the use of this platform, makes it possible of them to design robotic videography projects.

\section{ROBOTIC MARIONETTE CAMERA (RMC) PLATFORM COMPONENTS}

Robotic Marionette Camera (RMC), is a cyberphysical platform - hardware interface and software driver, that aims to simplify the user interface for robotic videography through physical interaction.

As a cyberphysical platform, RMC is made out of multiple core components to enable data communication, interactivity, and the videography process. These components include: 1- core software, 2- microcontroller (processor), 3- sensor based handels, 4-multisensory input, 5- housing, and 6- Robot arm (Figure 1).

\section{COMPONENT 01: CORE SOFTWARE}

To connect the physical controller to the processor of the robot, it is needed to have a software component to serve as a bridge.

RMC as a platform that is intended to be used by users with limited technical background, it was crucial that software operates in the background and as a backend part of the platform. However, to make it easier for users with technical/programming skills to modify some parts of the 
software, the central core software, and communication bridge is developed in Grasshopper 3D within Rhino 3D software.

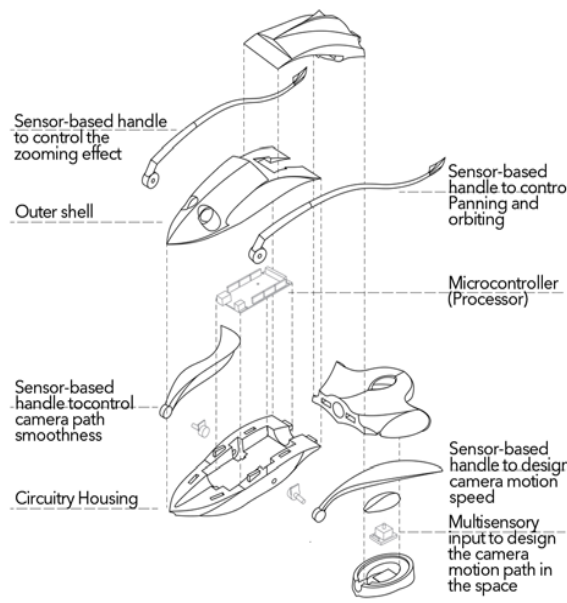

Figure 1: Some of the RMC hardware componentes

Using Transmission Control Protocol (TCP) communication, through Grasshopper 3D and by providing the static IP of the robot computer, Grasshopper 3D environment, and Robot computer can communicate via TCP data sending-receiving. Receiving data from the robot-its position and orientation and the possibility of sending data to change the robot's position, connects the world of digital data and physical performance.

To connect and simplify the steps for the physical controller, and to design the motion in a more intuitive way, we employed a custom-made animation-based plugin for Grasshopper 3D Oriole (Poustinchi, 2019). This plug-in simplifies the robotic motion design process into a simple animation based frame design where it is much easier to modify and design robotic motion with limited inputs (Figure 2).

Using the power of Oriole plugins, this platform has been used to develop different videography operations, including physical zooming (dolly and truck), rotating (panning, and tilting), pedstal, rolling and general movement and aiming, as presets for the controller.

\section{COMPONENT 02: MICROCONTROLLER (PROCESSOR)}

To communicate the date from physical/analog sensors, process them, and send them to the software core, there is a need for a processor unit: a microcontroller. Considering the ease of use and data communication through simple connectors-USB connection or basic Bluetooth, Arduino as a microcontroller of choice stood out immediately.

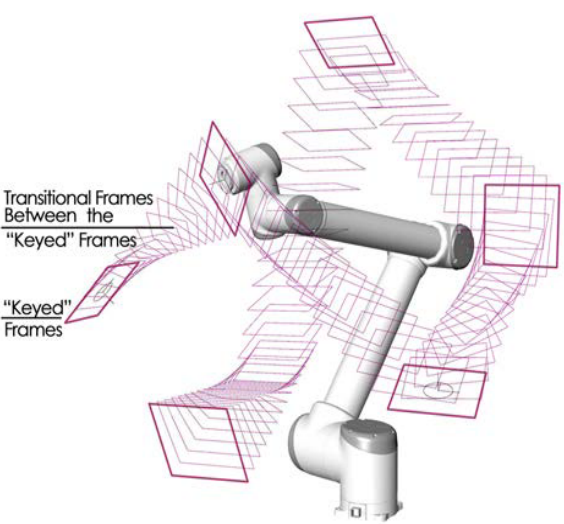

Figure 2: Developing a motion path animation for a UR-10 robot arm, based on a limited number of planes and inputs using [Hidden for Peer Review] plugin.

In this project, an Arduino Mega 2560 microcontroller has been used due to its capability to receive up to 16 analog inputs.

\section{COMPONENT 03: SENSOR BASED HANDELS}

To receive data from the physical environment, RMC is equipped with multiple resistance-based analog sensors. Using rotary potentiometer sensors, each of the handles can produce a range of numbers with 1024 steps.

Each of the senesors are controller by a "handle" that makes it easier for the user to modify the input of the sensor. There are a totall of four senesor based handels and each controll a specific videography task:

1- Zoom Handel: to move the camera forward-backward (dolly) and to the right and left (truck). Using the presets from the core software, zoom handle enables users to design linear cinematic motions.

2- Orbit Handel: to rotate the camera through panning or rolling.

3- Smoothness Handel: to control the number of frames between main positions of the camera, as a way to smooth the motion path.

4- Speed Handel: to adjust the speed of the motion-from a frame to another and overall rate. This handle enables the user to adjust, increase, or decrease the motion's speed while designing the action or when operating the designed motion.

\section{COMPONENT 04: MULTISENSORY INPUT}

Serving as a dynamic joystick and a swith, multisensory input component enables the user to record, modify and adjust the designed frames.

Using all the sensor-based handels, RMC users can develop the position/orientation for the camera motion as a frame in the Oriole plug-in in Grasshopper 3D. To be able 
to record these frames as a sequence for motion design, multisensory input serves as a middle component to enable the user to record the frames, mover through them, and modify them. In another word, this component serves as an indexing mechanism to organize the frames and put them in order.

\section{COMPONENT 05: HOUSING}

In order to collect all the physical parts and components of RMC in a user-friendly combination, the housing component has been used. This 3D-printed component serves as a receiving hub for the microcontroller, and all the sensors through custom-designed ducts for each component.

Precisely designed as a physical interface, the housing component becomes the leading presence of the RMC physical interface (figure 3 and 4).

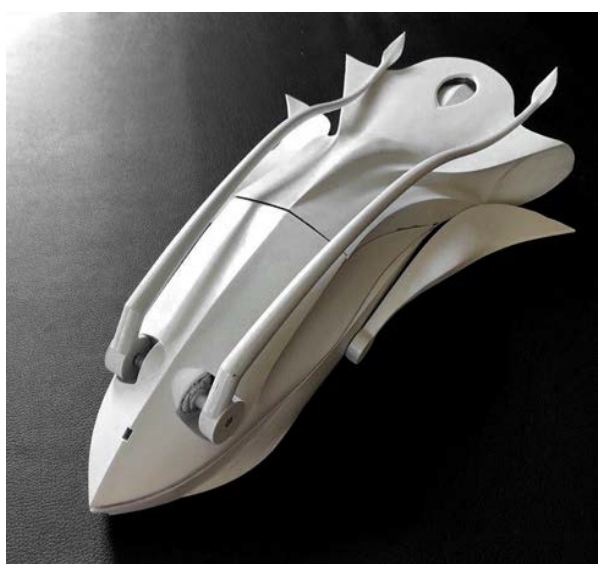

Figure 3: Physical RMC components housed in a 3D-printed housing shell and working as one unit-physical interface.

\section{COMPONENT 06: ROBOT ARM}

To complete the RMC platform and experience, there is a need for a robot arm. Although RMC is adaptable to a variety of robot brands, for the testing purposes and in this article, it has been tested using a UR-10, Universal Robot.

Know as "collaborative robots", and due to safety conciderations, Universal Robots were the best choice to test RMC platform for designers with limited technical background and experience with robotics.

\section{ROBOTIC MARIONETTE CAMERA (RMC) PLATFORM OPERATION AND RESULTS}

Although the purpose of this article is to report on RMC development and its conceptual and technical details, as a way to evaluate RMC platform's functionality, it has been tested in operation as part of the RAD Lab. Explored as a part of a design studio at Kent State University, students with different design and technical

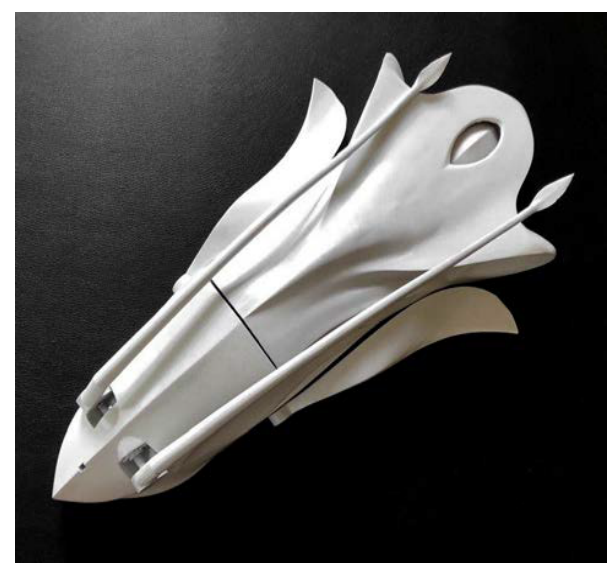

Figure 4: Top view of the RMC hardware controller.

background has been asked to use RMC to develop robotic videography of their physical models. Although most of these selected groupd of eleven students had some familiarity with digital camera and animation, this experience was their first attempt to design a physical animation/videography with a robot.

By connecting the RMC hardware interface to the core software component, students immediately started to intuitively learn the impact of each analog input on the controller. Very similar to controlling a Marionette toy, each student used the sensor-based handles to evaluate the robotic motion and to adjust the exact position of the robot end-effector, to make it ready for mounting the camera (Figure 5).

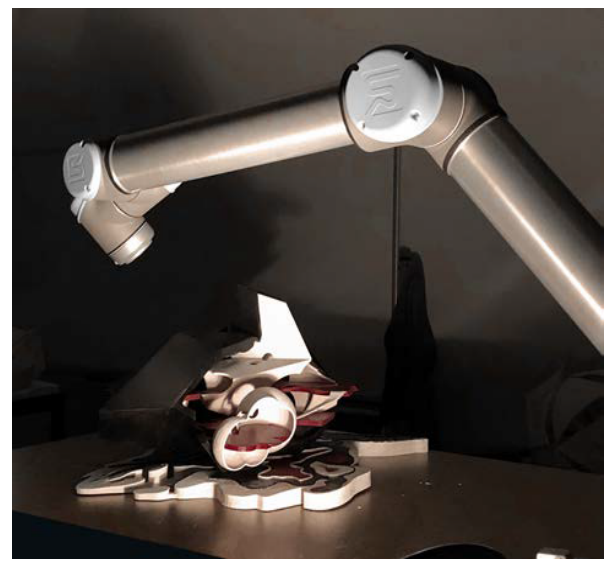

Figure 5: Adjusting the position of the robot end-effector-to receive the camera, using $\mathrm{RMC}$ platform and the physical controller.

Using a back and forth process, recording, and adjusting the frames through the multisensory input component, users were able to create a sequence of frames based on the camera's desired angles in relation to the physical model. 
Deciding on point-to-point movements of the robotpolyline motion, and the smoothness of the other transitions-spline motion, through the use of smoothness handle, the transitional logic between frames was generated..

Combining the generated frames into a single camera motion path, RMC enabled users to produce robotic videography path based on desired camera motion/position/orientation (Figure 6).

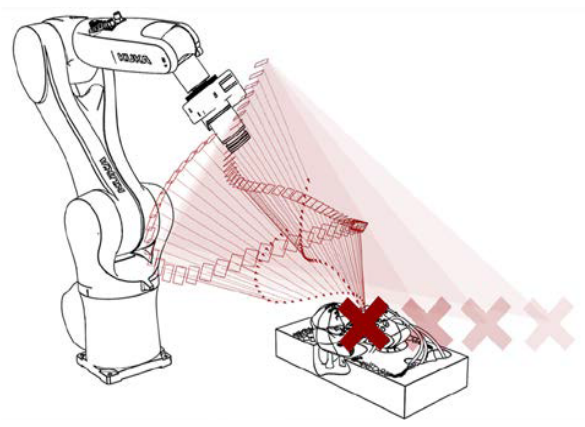

Figure 6: RMC core software combines the recorded frames into one motion path for the robotic camera.

Initial Comparison of the results of the digitally designed camera-paths--using limited programming skills, and the ones that have been developed using RMC physical interface, it was observed, that using RMC platform, users with limited design and technical skills were able to create a robotic videography camera-path in a more intuitive way. It was also observed that the use of a physically interactive interface to design robotically controlled camera-paths, as well as seeing the results in the physical world immediately, users were able and willing to re-iterate camera-path much faster compering to a fully digital design process.

\section{DISCUSSION}

$\mathrm{RMC}$ is a part of a more significant body of research looking at the human-computer and human-robot interaction. In its first iteration-presented in this paper, RMC seeks possibilities of an intuitive tangible user interface as a possible method of interaction with robotic technology in a more intuitive way. This research hopes to become a platform to increase user engagement and diversify user groups by simplifying the robotic motion design process through a physical interface.

Given the presented focus of RMC, this article aimed to illustrate RMC as a platform without necessarily evaluating its tangible impact since that would take more experimentation and evaluation. However, one of the limitations of the current research project is the limited number of users-eleven studetns, and the similarity of their background. For the second phase of the project, we aim to make RMC available to a bigger group of users with more diverse design and technical education. It is also intended for the next phase of the project to directly compare the results of a programmed robotic motion and an RMC developed camera path, to contract the differences and possible advantages of each method.

\section{ACKNOWLEDGMENTS}

RMC project has been developed and funded as part of the Robotically Augmented Design (RAD) Lab at the College of Architecture and Environmental Design at Kent State University.

\section{REFERENCES}

Braumann, J. and Brell-Cokcan S. 2011. "Parametric Robot Control." Proceedings of the 31st Annual Conference of the Association for Computer Aided Design in Architecture (ACADIA) 242-51.

Braumann, J. and Brell-Cokcan S. 2012. "Real-Time Robot Simulation and Control for Architectural Design." Digital Physicality Proceedings of the 30th ECAADe Conference 2:479-86.

Brugnaro G., Baharlou E., Vasey L., Menges A. 2016. "Robotic Softness: An Adaptive Robotic Fabrication Process for Woven Structures." In: Proceedings of the 36th Annual Confer-ence of the Association for Computer Aided Design in Architecture (ACADIA), Ann Arbor, MI, USA. 154-163.

Budig, M., Lim J., and Petrovic R.. 2014. "Integrating Robotic Fabrication in the Design Process." Architectural Design 84(3): 22-43.

Gramazio, F., Matthias K., and Jan W.. 2008. "Authoring Robotic." Architectural Design 3(84):14-22.

Gramazio, F., Matthias K., and Jan W.. 2008. "Authoring Robotic." Architectural Design 3(84): 14-22.

Kruysman, B. and Proto J. 2014, "Impossible objects," Architectural Design 227, edited by B. Sheil.106-111.

Lynn, G. 2008, "Robots," in Greg Lynn Form, ed. Mark Roppolt, New York, NY: Rizzoli International Publication Inc., 251-278.

Nof S. Y. 1999. 1 Handbook of Industrial Robotics Vol. 1. John Wiley \& Sons.

Ozel, G. 2016 “Toward a postarchitecture," Log36, 99-105

Ozel G., 2015 "Case for an Architectural Singularity: Synchronization of Robotically Actuated Motion, Sense-Based Interaction and Computational Interface," In Proceedings of the 34th Annual Conference of the Association for Computer Aided Design in Architecture (ACADIA), edited by D. Gerber, A. Huang, and J. Sanchez, (Los Angeles, CA: ACADIA/ Riverside Architectural Press), 399-408.

Payne, A. 2011. "A Five-Axis Robotic Motion Controller for Designers." Integration Through Computation - Proceedings of the 31st Annual Conference of the Association for Computer Aided Design in Architecture, ACADIA 2011 162-69.

Poustinchi, E. 2019, "Robotically Augmented Imaging (RAI Alpha)" In ACADIA 19:UBIQUITY AND AUTONOMY [Proceedings of the 39th Annual Conference of the Association for Computer Aided Design in Architecture (ACADIA) ISBN 978-0-57859179-7] (The University of Texas at Austin School of Architecture, Austin, Texas 21-26 October, 2019) pp. 352-359

Poustinchi, E. 2019, "Oriole Beta - A Parametric Solution for Robotic Motion Design Using Animation". Sousa, JP, Xavier, JP and Castro Henriques, G (eds.), Architecture in the Age of the 4th Industrial Revolution - Proceedings of the 37th eCAADe and 23rd SIGraDi Conference - Volume 2, University of Porto, Porto, Portugal, 11-13 September 2019, pp. 227-234

Prado, M, Dorstelmann M.,Schwinn T., Menges A., and Knippers J.. 2014. "Core-Less Filament Winding." in Robotic Fabrication in Architecture, Art and Design 2014, edited by W. McGee and M. Ponce De Leon. Springer. 
Retsin, G. and Manuel J. G.. 2016. "Discrete Computational Methods for Robotc Additive Manufacturing." ACADIA 2016: Posthuman Frontiers: Data, Designers, and Cognitive Machines - Proceedings of the 36th Annual Conference of the Association for Computer Aided Design in Architecture 33241.

Schwartz, T. 2013. "HAL." In Rob| Arch 2012, eds. Sigrid BrellÇokcan and Johannes Braumann. Springer-Verlag Wien, 92-101.

Soler V., Retsin G., Jimenez M. G. 2017, "A Generalized Approach to Non-Layered Fused Fil-ament Fabrication". In: Proceedings of the 37th Annual Conference of the Association for Computer Aided Design in Architecture (ACADIA), Cambridge, MA, USA, 562- 571.

Testa, P. 2017. "Imaging," in Robot House, edited by P. Testa, New York, NY: Thames \& Hudson Inc., 155-219.

Vasey, L., Grossman T., Nagy D., Kerrick H., Atherton E., Thomasson D., Cote N., Schwinn T., Benjamin D., Conti M., Fitzmaurice G., and Menges A.. 2016. "Collaborative Construction: Human and Robot Collaboration Enabling the Fabrication and Assembly of a Filament-Wound Structure." Pp. 184-95 in
Wiscombe, T. 2014, "Discreteness or towards a flat ontology of architecture." Project, (Issue 3), 34-43.

Yunis L., Kyjánek O., Dörstelmann M., Prado M., Schwinn T. and Menges A. 2014, "Bio-inspired and fabrication-informed design strategies for modular fibrous structures in architecture, Fusion." In: Proceedings of the 32nd eCAADe Conference, Publisher, Newcastle upon Tyne, England, UK, 423-432.

Websites:

"Bot \& Dolly Fuses 3D Animation and Industrial Automation": 2013. Retrieved from robotics business review <https://www.roboticsbusinessreview.com/consumer/bot doll y_fuses_3d_animation_and_industrial_automation/> (accessed 2 th, July 2020).

Munkowitz, B. G. "BOX". Gmunk, Sep. 2013, Retrieved from

< https://gmunk.com/BOX> (Accessed 2 July 2020.)

Retrieved from <http://motorizedprecision.com/> (Accessed 2th, July 2020.) 